\title{
Flowering-time genes modulate meristem determinacy and growth form in Arabidopsis thaliana
}

\author{
Siegbert Melzer ${ }^{1}$, Frederic Lens ${ }^{2,3}$, Jerôme Gennen ${ }^{1}$, Steffen Vanneste ${ }^{1}$, Antje Rohde ${ }^{1}$ \& Tom Beeckman ${ }^{1}$
}

Plants have evolved annual and perennial life forms as alternative strategies to adapt reproduction and survival to environmental constraints. In isolated situations, such as islands, woody perennials have evolved repeatedly from annual ancestors $^{1}$. Although the molecular basis of the rapid evolution of insular woodiness is unknown, the molecular difference between perennials and annuals might be rather small, and a change between these life strategies might not require major genetic innovations ${ }^{2,3}$. Developmental regulators can strongly affect evolutionary variation ${ }^{4}$ and genes involved in meristem transitions are good candidates for a switch in growth habit. We found that the MADS box proteins SUPPRESSOR OF OVEREXPRESSION OF CONSTANS 1 (SOC1) and FRUITFULL (FUL) not only control flowering time, but also affect determinacy of all meristems. In addition, downregulation of both proteins established phenotypes common to the lifestyle of perennial plants, suggesting their involvement in the prevention of secondary growth and longevity in annual life forms.

Plant growth originates from a small number of undifferentiated cells called meristems. Primary meristems are established during embryogenesis and form primary tissues from which all plant organs develop. Secondary meristems, such as axillary meristems and the cambium, originate within primary tissues. Meristems can be determinate-that is, consumed for the formation of an organ-or indeterminate, meaning that they are active throughout the life span of a plant. Upon floral induction in annual plants, vegetative shoot meristems undergo the transition to inflorescence meristems. These inflorescence meristems will remain indeterminate for some time to generate determinate floral meristems giving rise to flowers. Finally, all meristems are consumed and the plants die in the same growing season. In contrast, perennial plants have evolved more elaborate life strategies to survive harsh environmental conditions for many years by forming perennial structures such as overwintering buds, bulbs or tubers, which contain at least one indeterminate meristem for the outgrowth in the next season ${ }^{2}$. Often, perennial plants incorporate enormous amounts of long-lived and eventually dead biomass (wood) through cambial activity (secondary growth).

Arabidopsis thaliana is a small annual herb in which floral induction is controlled by different flowering-time pathways. These pathways depend on environmental cues, such as day length (photoperiod) and vernalization (cold temperature), or on plant age. Arabidopsis is a facultative long-day plant that flowers much faster under long $(16 \mathrm{~h} /$ day) than short $(8 \mathrm{~h} /$ day $)$ light periods. After perceiving flowering-inducing long days, the key regulator of the photoperiodic pathway, CONSTANS (CO), activates FT (FLOWERING LOCUS T) in the leaf vasculature. The FT protein is transported to apical meristems, where it triggers the floral transition ${ }^{5}$. SOC1 (AGL20) and FUL (AGL8) are MADS box genes acting downstream of FT in apical meristems, but they are already expressed in leaves-independently of FT-during the vegetative phase. Early upon floral induction SOC1 and FUL are induced in apical meristems, and, later on, both genes are also expressed in procambial strands of developing inflorescences $^{6-11}$. FUL has been described for its role in fruit dehiscence ${ }^{12}$,

Table 1 Flowering time, assayed by leaf numbers of the basal rosette

\begin{tabular}{|c|c|c|c|c|c|c|c|c|}
\hline \multirow[b]{2}{*}{ Genotype } & \multicolumn{4}{|c|}{ Long days } & \multicolumn{4}{|c|}{ Short days } \\
\hline & $\mathrm{RL}$ & $\mathrm{CL}$ & $\mathrm{TL}$ & s.d. & $\mathrm{RL}$ & $\mathrm{CL}$ & TL & s.d. \\
\hline Col & 12.1 & 3.0 & 15.1 & 1.4 & 60.7 & 9.9 & 70.6 & 2.5 \\
\hline ful-2 & 13.8 & 5.6 & 19.4 & 1.3 & 62.9 & 12.7 & 75.6 & 3.6 \\
\hline $\operatorname{soc} 1-3$ & 23.6 & 4.4 & 28.0 & 2.2 & 82.7 & 7.9 & 90.6 & 2.5 \\
\hline $\operatorname{soc} 1-3$ ful-2 & 34.1 & 5.2 & 39.3 & 1.1 & 83.1 & 11.8 & 94.8 & 2.9 \\
\hline 35S:AGL19 & 3.9 & 3.5 & 7.5 & 0.8 & 11.8 & 5.7 & 17.5 & 0.8 \\
\hline 35S:AGL19 soc $1-3$ ful-2 & 4.2 & 10.9 & 15.1 & 1.4 & 11.7 & 7.3 & 19.0 & 1.6 \\
\hline 35S:FT & 3.4 & 1.4 & 4.9 & 0.8 & 3.5 & 1.8 & 5.2 & 0.9 \\
\hline 35s:FT ful-2 & 7.9 & 2.5 & 10.4 & 0.9 & 8.5 & 2.4 & 10.9 & 1.3 \\
\hline 35S:FT soc 1-3 & 9.4 & 0.5 & 9.9 & 1.1 & 9.3 & 1.2 & 10.5 & 1.2 \\
\hline 35S:FT soc $1-3$ ful-2 & 21.6 & 4.5 & 26.1 & 1.3 & 32.2 & 8.2 & 40.4 & 1.9 \\
\hline
\end{tabular}

$\mathrm{RL}$, rosette leaves; $\mathrm{CL}$, cauline leaves; TL, total leaves; s.d., standard deviation of the mean.

\footnotetext{
${ }^{1}$ Department of Plant Systems Biology, Flanders Institute for Biotechnology and Department of Molecular Genetics, Ghent University, Technologiepark 927 , B-9052 Ghent, Belgium. ${ }^{2}$ Laboratory of Plant Systematics, Institute of Botany and Microbiology, Katholieke Universiteit Leuven, Kasteelpark Arenberg 31 , B-3001 Leuven, Belgium. ${ }^{3}$ National Herbarium of the Netherlands, Leiden University Branch, P.O. Box 9514, NL-2300 RA Leiden, The Netherlands. Correspondence should be
} addressed to S.M. (siegbert.melzer@psb.ugent.be). 

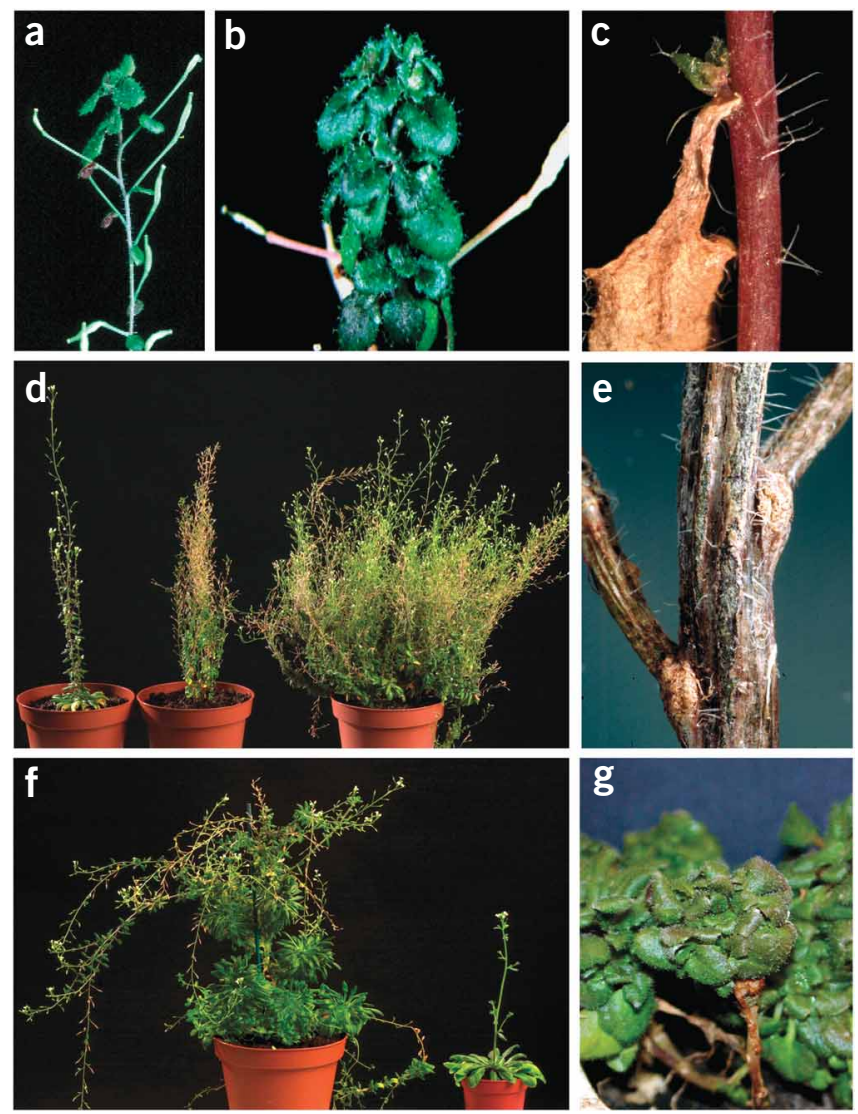

but as SOC1 and FUL also interact in yeast two-hybrid experiments as homo- and heterodimers ${ }^{13}$, FUL might have additional functions that may be in part redundant to those of SOC1 in areas of overlapping expression. The soc1 mutants flower significantly later in long and short days, whereas ful mutants are only slightly delayed ${ }^{7-9,12}$ (Table 1). Flowering in double mutants with different combinations of $\operatorname{soc} 1$ and $f u l$ mutant alleles was further delayed only in long-day conditions. In short days, the double mutants flowered no later than soc1 single mutants (Table 1 and Supplementary Fig. 1a,b online), suggesting that both genes might have a redundant role for photoperiodic control of flowering time.

Figure 2 Secondary growth in soc1-3 ful-2 plants. (a) Hand section through a 5-mm-thick lateral branch of a 5-month-old double mutant with a secondary xylem stained dark blue and a secondary phloem stained pink. The inset shows a wild-type main stem at the same magnification. (b) Magnified cross-section through a 5-mm-thick lateral branch of a 5-month-old double mutant showing radial files of xylem and phloem derived from secondary cambium (on the line between yellow arrowheads). (c) Crosssection through the base ( $1 \mathrm{~cm}$ above the rosette) of a 6 -cm-long wild-type inflorescence showing only primary tissues in the marked area. socl and ful single mutants did not show secondary growth and were indistinguishable from wild type. (d) Cross-section through a soc1-3 ful-2 inflorescence at the same developmental stage as the wild-type plant in c, with files of cells of secondary xylem and phloem (additional cell layers in the marked area). $\mathrm{QJ} ;$ At this stage the interfascicular cambium was initiated and fused with QJ;the cambium from the vascular bundles to form a closed cambium. (e) Secondary growth in a 6-cm-long 35S:AGL 19 soc1-3 ful-2 inflorescence stem $1 \mathrm{~cm}$ above the rosette with secondary xylem and phloem. (f) Massive secondary growth in the inflorescence stem of a 5-month-old 35S:AGL19 soc1-3 ful-2 plant $1 \mathrm{~cm}$ above the rosette. Scale bars in a, $500 \mu \mathrm{m} ; \mathbf{c}, \mathbf{d}, \mathbf{f}$, $100 \mu \mathrm{m} ; \mathrm{e}, 200 \mu \mathrm{m}$. SP, secondary phloem; SX, secondary xylem.
Figure 1 Perennial features in soc1-3 ful-2 mutants. (a) Floral reversion at the top of a soc1-3 ful-2 inflorescence. (b) soc 1-3 ful-2 apical rosettes at a later stage than shown in a. (c) Dead basal cauline leaf with a small arrested axillary shoot that will develop into an aerial rosette in the next growth wave. (d) Comparison of soc 1-3 ful-2 mutants grown in long days for 6 weeks (left), 3 months (middle) or 5 months (right). (e) Woody stem of a 4-monthold soc 1-3 ful-2 mutant in long days. (f) Comparison of an 8-month-old soc 1-3 ful-2 mutant with many aerial rosettes at the base and floral reversion at inflorescence meristems (left) and a 2-month-old wild-type plant (right) grown in short days. (g) A 14-month-old soc1-3 ful-2 mutant with rosettes on lateral branches.

During seed ripening in long days, many apically positioned inflorescence meristems of soc1-3 ful-2 mutants reverted to a vegetative state, producing small true leaves with axillary meristems in apical rosettes (Fig. 1a,b and Supplementary Fig. 2a online), which had never been observed before in Arabidopsis ${ }^{14}$. Meristems in cauline leaf axils at the base of double-mutant inflorescences stayed in a vegetative phase and developed aerial rosettes (Supplementary Fig. 2b). Additionally, the double mutants formed bracts that are normally absent in Arabidopsis $^{15}$ (Supplementary Fig. 3 online). Wild-type and singlemutant plants senesced and died after seed maturation. In all double mutants, basal rosette leaves, cauline leaves and siliques senesced as well, whereas the apical rosettes resulting from inflorescence meristem reversions, the aerial rosettes and the stems remained alive. Of note, arrested vegetative shoots resembling dormant buds persisted in the axils of dead cauline leaves (Fig. 1c). These buds and also the aerial and apical rosettes grew out to form new inflorescences and rosettes in a next growth cycle (Supplementary Fig. 2c). Subsequently, several distinct waves of growth occurred, where again inflorescence meristems reverted to vegetative meristems and aerial rosettes formed at the base of the inflorescences. The socl ful double mutants developed into highly branched shrubs in both ecotypes tested (Fig. 1d and Supplementary Fig. 4 online). In short days, socl ful mutants presented no distinct growth cycles and produced fewer inflorescences, but again with reversions of inflorescence meristems. Nevertheless,
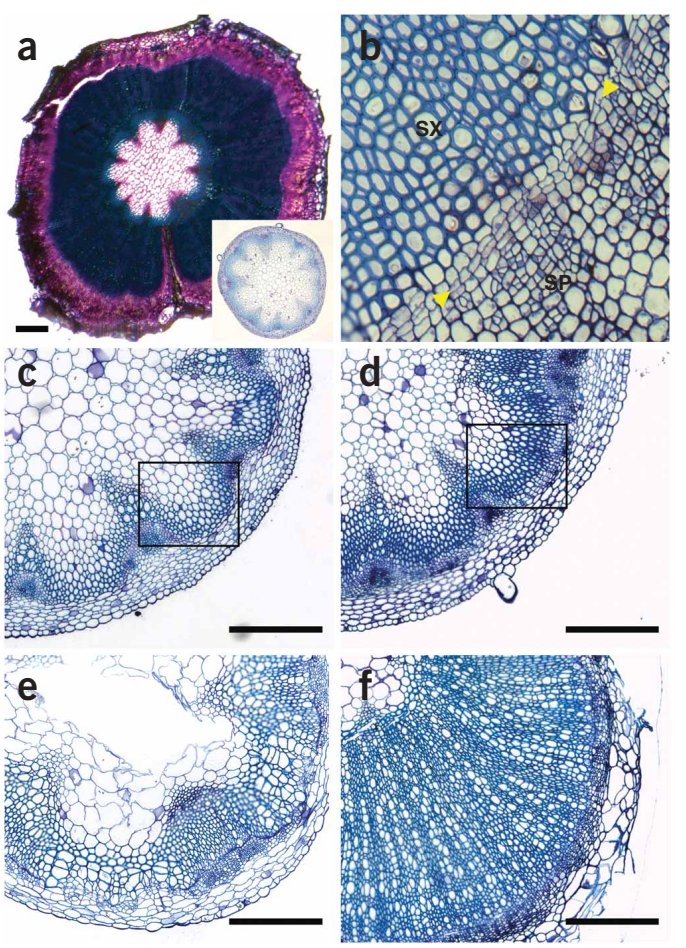

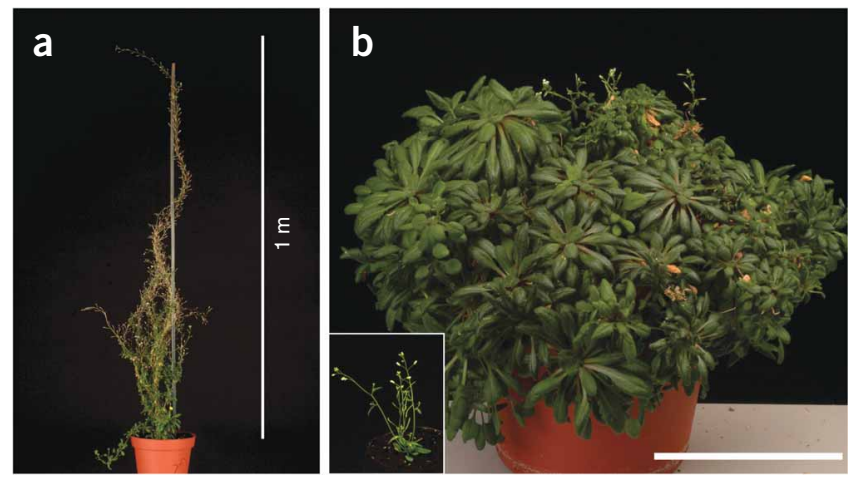

Figure 3 Different plant growth forms in soc 1-3 and ful-2 mutants.

(a) A ft-1 ful-2 double mutant with an exaggerated indeterminate growth (b) A 35S:FT soc 1-3 ful-2 plant showing a cushion-plant growth habit after 8 months of growth in short days. The inset shows a 35S: FT plant grown in short days. Scale bar, $10 \mathrm{~cm}$.

they showed a markedly increased life span and a shrub-like phenotype through the continuous growth of vegetative aerial rosettes (Fig. 1f,g and Supplementary Fig. 2d). In contrast to wild-type plants, which showed only limited secondary growth in inflorescence stems $^{16}$, soc1 ful mutants readily developed woody stems under either photoperiod (Fig. 1e and Supplementary Fig. 2e). The extensive secondary growth finally formed a considerable wood cylinder that had never been observed before in Arabidopsis (Fig. 2a,b).

To ascertain whether secondary growth resulted from the loss of both SOC1 and FUL or merely from the increased longevity, we analyzed the initiation of secondary growth. Wild-type and soc1-3 ful-2 plants were grown in short days to a stage that allowed immediate floral induction after transfer of the plants to long days. In soc1-3 ful-2 mutants, a closed cambium was established at a very early stage of inflorescence stem development. Secondary growth was already evident in the basal part of young 6-cm-long socl ful inflorescences, and this growth gradually extended into the upper part of the inflorescence stem. No signs of such secondary growth could be recorded in wild-type plants at comparable stages (Fig. 2c,d). At a later developmental stage, all soc1-3 ful-2 stems, including those of higher-order co-inflorescences, had steadily increasing amounts of secondary tissues (Supplementary Fig. 5 online). The early initiation of a cambium in inflorescence stems of socl ful mutants indicates that the loss of SOC1 and FUL function rather than the increased life span of the plants was responsible for the observed secondary growth. Recently it was reported that floral induction is a condition for xylem expansion in hypocotyls and roots ${ }^{17}$ at stages before inflorescences are formed. However, whether SOC1 and FUL activity also have to be modulated in these organs is not yet known.

To test further whether late flowering was a prerequisite for the observed extreme longevity and secondary growth, we overexpressed the flowering-time regulator AGL19 (AGAMOUS LIKE 19) under the control of the CaMV 35 S promoter in soc1-3 ful-2 mutants. AGL19 controls flowering downstream of a cold-perception pathway and acts independently of FT and SOC1 (ref. 18); therefore, it might uncouple flowering from longevity and secondary growth. Indeed, the 35S:AGL19 transgene caused the soc1-3 ful-2 double mutants to flower as early as wild-type plants containing the 35S:AGL19 transgene (Table 1). Irrespective of the extremely early flowering in long days (Supplementary Fig. 6a-c online), 35S:AGL19 soc1-3 ful-2 plants showed indeterminate growth, recurrent growth cycles and the development of small aerial rosettes that formed many co-inflorescences reminiscent of soc1-3 ful-2 plants (Supplementary Fig. 6d-f). Moreover, secondary growth was already visible in very young stems that steadily increased in diameter as a result of the significant production of secondary tissues (Fig. 2e,f). Secondary growth and longevity were established independently of pleiotropic effects of late flowering and can be attributed to the loss of SOC1 and FUL in the various soc1 ful mutant combinations. Thus, SOC1 and FUL not only control flowering time, but also play a key part in determining the herbaceous growth form and the short life cycle of Arabidopsis.

Investigating whether additional flowering time genes might be equally implicated in the adoption of perennial characteristics, we found that FT additionally modified the growth form of soc1-3 and ful-2 mutants. The $\mathrm{ft}-1$ mutants flower late only in long days, whereas the 35S:FT transgene triggers an extremely early and photoperiodindependent flowering in wild type $\mathrm{e}^{19,20}$. The socl and ful single mutations slightly delayed the strong early flowering in 35S:FT plants $^{21,22}$ (Table 1). Despite the very early flowering of 35S:FT soc1-3 and 35S:FT ful-2 plants, they showed a markedly increased longevity and produced through reiteration of growth many short determinate co-inflorescences, particularly in short days (Supplementary Fig. $\mathbf{6 g}, \mathbf{h}$ ). These results suggest that the loss of SOC1 or FUL activity is sufficient to increase the lifespan of plants independent of flowering time. Combination of the $f t-1$ mutation with ful-2 led to an exaggerated indeterminacy of the apical meristem, with plants reaching a height of up to $1 \mathrm{~m}$ and developing only a few side branches (Fig. 3a). Finally, the inflorescence meristems of the $f t-1$ ful-2 double mutants also reverted to vegetative growth. The loss of FT function in socl-3 mutants caused the formation of multiple rosettes during the vegetative phase and of large aerial rosettes during reproduction in short days (Supplementary Fig. 6i). As these phenotypes resembled those of soc1-3 ful-2 double mutants, either FT (loss of FT including the downregulation of its targets SOC1 or FUL) or proteins downstream of FT act redundantly with SOC1 and FUL to prevent indeterminate growth, floral reversion and aerial rosette formation. These results suggest that FT-besides triggering flowering-also regulates the fate of meristems and consequently affects growth, especially under short days. That FT also regulates growth processes other than flowering has also been shown in tomato ${ }^{23}$.

Notably, the combined socl-3 ful-2 mutations suppressed early flowering in 35S:FT plants synergistically (Table 1), indicating that SOC1 and FUL have redundant and clearly more important roles in flowering-time control by day length downstream of FT than previously anticipated. 35S:FT soc1-3 ful-2 mutants developed a short main inflorescence and few co-inflorescences that all terminated early. Again, reversions of inflorescence meristems and aerial rosettes at the base of the inflorescences allowed for the reiteration of vegetative growth. Many large rosettes formed at the bases of these co-inflorescences, generating a cushion-plant phenotype under short-day conditions. Most of the rosette meristems remained vegetative for many months (Fig. 3b), showing that constitutive expression of the mobile floral stimulus FT cannot provoke the transition from vegetative to generative meristems in the absence of SOC1 and FUL. Instead, an even more exaggerated vegetative long-lived phenotype developed, indicating again that not only FUL and SOC1, but also FT, have functions beyond flowering-time control.

In conclusion, the modulation of the activities of only three genes had a clear effect on indeterminacy of meristems and longevity of the plants, leading to the development of markedly different growth forms in Arabidopsis. Finally, aspects of the soc1-3 ful-2 double mutants, such as vegetative buds, recurrent growth cycles, longevity and extensive woodiness, are reminiscent of plants with a perennial life style. Similar 
phenotypic traits have also been acquired in Sy-0 (refs. 24,25), a naturally occurring Arabidopsis accession from the Isle of Skye. In Sy-0, the expression of SOC1 and FUL is strongly reduced (Supplementary Fig. 7 online).

In angiosperms, the perennial woody habit is believed to be the ancestral condition, from which annual herbaceous lineages have evolved several times independently. Conversely, evolution from annual herbaceous ancestors to perennial woody taxa has also repeatedly occurred $^{1}$. For example, in various annual herbaceous lineages, such as Sonchus and Echium, woody perennial species evolved on isolated islands from their continental annual ancestors ${ }^{26-28}$. Here we have shown the strong impact of only three genes (FT, SOC1 and FUL) on plant growth form and longevity in Arabidopsis. The rapid parallel evolution of 'insular woodiness' ${ }^{28}$ might also have been established through mutations in a small number of developmental genes.

\section{METHODS}

Seeds of Arabidopsis thaliana (L.) Heyhn. We obtained Columbia (Col-0) and Landsberg erecta (Ler) ecotypes as well as ful-1 and ful-2 mutants from the Nottingham Arabidopsis Stock Centre. Seeds of 35S:FT plants were provided by P.Wigge (John Innes Centre), seeds of the soc1-1 allele were provided by G. Coupland (Max Planck Institute for Plant Breeding Research) and seeds of soc1-2 in Col and Ler backgrounds by I. Lee (University of Seoul). The soc1-3 mutant has been described previously as agl20-3 (ref. 7). Plants were grown in soil, either under short-day ( $8 \mathrm{~h} \mathrm{light} / 16 \mathrm{~h}$ dark) or long-day conditions $(16 \mathrm{~h}$ light $/ 8 \mathrm{~h}$ dark) at $22{ }^{\circ} \mathrm{C}$ under fluorescent tubes emitting a photon flux density of $150 \mu \mathrm{mol} \mathrm{m} \mathrm{m}^{-2} \mathrm{sec}^{-1}$. We assayed flowering time by counting leaf number. Reciprocal crosses of soc1-3 and ful-2, soc1-2 and ful-2 in Col backgrounds and soc 1-1 and socl-2 with ful-1 in Ler backgrounds generated identical phenotypes in the homozygous progenies. We monitored the ful-1 and ful-2 mutations by the silique phenotype and we genotyped the socl mutations by PCR.

For RT-PCR, we isolated total RNA from lower stem parts of Col-0 and Sy-0 plants and prepared cDNA as previously described ${ }^{7}$. Quantitative real-time PCR was run on an iCycler (BioRad). As a control, a fragment from the gene encoding the eukaryotic protein synthesis initiation factor $4 \mathrm{~A}$ (eIF4A) was amplified and used to normalize the data (Supplementary Table 1 online).

For microscopy, stem pieces of $3-5 \mathrm{~mm}$ were fixed overnight in $4 \%$ formaldehyde in $50 \mathrm{mM}$ phosphate ( $\mathrm{pH}$ 7). The stems were dehydrated in a graded ethanol series and transferred to Technovit 7100 (Kulzer) or LR white (medium grade) (London Resin) embedding medium according to the supplier instructions. Sections of $6-8 \mu \mathrm{m}$ were cut with a rotary microtome and stained with toluidine blue before viewing under a Leica microscope.

\section{Note: Supplementary information is available on the Nature Genetics website.}

\section{ACKNOWLEDGMENTS}

We appreciate the continuous support of K. Apel, D. Inzé and E. Smets. We thank J. Chandler and M. De Cock for critical reading of the manuscript. S.V. is indebted to the Institute for the Promotion of Innovation through Science and Technology in Flanders for a predoctoral fellowship. F.L. and A.R. are postdoctoral fellows of the Research Foundation-Flanders (FWO). Seeds of 35S:FT plants were provided by P. Wigge (John Innes Centre), seeds of the soc1-1 allele were provided by G. Coupland (Max Planck Institute for Plant Breeding Research) and seeds of socl-2 in Col and Ler backgrounds by I. Lee (University of Seoul).
Published online at http://www.nature.com/naturegenetics/

Reprints and permissions information is available online at http://npg.nature.com/ reprintsandpermissions/

1. Carlquist, S. Island Biology (Columbia University Press, New York, 1974).

2. Thomas, H., Thomas, H.M. \& Ougham, H. Annuality, perenniality and cell death. J. Exp. Bot. 51, 1781-1788 (2000).

3. Battey, N. \& Tooke, F. Molecular control and variation in the floral transition. Curr. Opin. Plant Biol. 5, 62-68 (2002).

4. Cronk, Q.C.B. Plant evolution and development in a post-genomic context. Nat. Rev. Genet. 2, 607-619 (2001).

5. Corbesier, L. et al. FT protein movement contributes to long-distance signalling in floral induction of Arabidopsis. Science 316, 1030-1033 (2007).

6. Wigge, P.A. et al. Integration of spatial and temporal information during floral induction in Arabidopsis. Science 309, 1056-1059 (2005).

7. Borner, R. et al. A MADS domain gene involved in the transition to flowering in Arabidopsis. Plant J. 24, 591-599 (2000).

8. Samach, A. et al. Distinct roles of CONSTANS target genes in reproductive development of Arabidopsis. Science 288, 1613-1616 (2000).

9. Lee, $\mathrm{H}$. et al. The AGAMOUS-LIKE 20 MADS domain protein integrates floral inductive pathways in Arabidopsis. Genes Dev. 14, 2366-2376 (2000).

10. Schmid, M. et al. Dissection of floral induction pathways using global expression analysis. Development 130, 6001-6012 (2003).

11. Hempel, F.D. et al. Floral determination and expression of floral regulatory genes in Arabidopsis. Development 124, 3845-3853 (1997).

12. Ferrandiz, C., Gu, Q., Martienssen, R. \& Yanofsky, M. Redundant regulation of meristem identity and plant architecture by FRUITFULL, APETALA1 and CAULIFLOWER. Development 127, 725-734 (2000).

13. de Folter, S. et al. Comprehensive interaction map of the Arabidopsis MADS Box transcription factors. Plant Cell 17, 1424-1433 (2005).

14. Tooke, F. \& Ordlidge, M. Chiurugwi, T. \& Battey, N. Mechanisms and function of flower and inflorescence reversion. J. Exp. Bot. 56, 2587-2599 (2005).

15. Long, J. \& Barton, M.K. Initiation of axillary and floral meristems in Arabidopsis. Dev. Biol. 218, 341-353 (2000).

16. Demura, T. \& Fukuda, H. Transcriptional regulation in wood formation. Trends Plant Sci. 12, 64-70 (2007).

17. Sibout, R., Plantegenet, S. \& Hardtke, C.S. Flowering as a condition for xylem expansion in Arabidopsis hypocotyls and root. Curr. Biol. 18, 458-463 (2008).

18. Schönrock, N. et al. Polycomb-group proteins repress the floral activator AGL19 in the FLC-independent vernalization pathway. Genes Dev. 20, 1667-1678 (2006).

19. Kardailsky, I. et al. Activation tagging of the floral inducer FT. Science 286, 1962-1965 (1999).

20. Kobayashi, Y. et al. A pair of related genes with antagonistic roles in mediating flowering signals. Science 286, 1960-1962 (1999).

21. Teper-Bamnolker, P. \& Samach, A. The flowering integrator FT regulates SEPALLATA3 and FRUITFULL accumulation in Arabidopsis leaves. Plant Cell 17, 2661-2675 (2005).

22. Yoo, S.K. et al. CONSTANS activates SUPPRESSOR OF OVEREXPRESSION OF CONSTANS 1 through FLOWERING LOCUS T to promote flowering in Arabidopsis. Plant Physiol. 139, 770-778 (2005).

23. Lifschitz, E. et al. The tomato FT orthologs triggers systemic signals that regulate growth and flowering and substitute for diverse environmental stimuli. Proc. Natl. Acad. Sci. USA 103, 6398-6403 (2006).

24. Poduska, B. Humphrey, T. Redweik, A. \& Grbic, V. The synergistic activation of FLOWERING LOCUS C by FRIGIDA and a new flowering gene AERIAL ROSETTE 1 underlies a novel morphology in Arabidopsis. Genetics 163, 1457-1465 (2003)

25. Wang, Q. et al. HUA2 caused natural variation in shoot morphology of $A$. thaliana. Curr. Biol. 17, 1513-1519 (2007).

26. Groover, A.T. What genes make a tree a tree? Trends Plant Sci. 10, 210-214 (2005).

27. Kim, S.-C., Crawford, D.J., Francisco-Ortega, J. \& Santos-Guerra, A. A common origin for woody Sonchus and five related genera in the Macaronesian islands: molecular evidence for extensive radiation. Proc. Natl. Acad. Sci. USA 93, 7743-7748 (1996).

28. Böhle, U.-R., Hilger, H.H. \& Martin, W.F. Island colonization and evolution of the insular woody habit in Echium L. (Boraginaceae). Proc. Natl. Acad. Sci. USA 93, 11740-11745 (1996). 